\title{
Data Acquisition and Processing System under Hydrogen Sensor
}

\author{
Shimin Wang, Yongjie Yang*, and Junjiao Zhang \\ School of Electronics and Information, Nantong University, Nantong, 226019, China
}

\begin{abstract}
To facilitate the data acquisition and analysis of hydrogen sensors in early stages, a set of data acquisition and processing systems for hydrogen sensors has been developed. The system applies the embedded technology and combines the acquisition terminal with the upper computer to realize the data acquisition and processing of hydrogen sensors. At present, it has been applied to a constant voltage type hydrogen sensor to collect and process data. The conversion from resistance to current to voltage is realized. The resistance value at both ends of the measured material is within $200 \mathrm{~K} \Omega$. The measurement accuracy is $1 \Omega$. The upper computer assists with the temperature compensation mechanism to achieve the effective data acquisition and processing of the measured materials. The practical application shows that the system is of great significance to the data acquisition and processing of early hydrogen sensors.
\end{abstract}

Keywords: hydrogen sensor; data acquisition; embedded technology

(Submitted on May 21, 2018; Revised on June 24, 2018; Accepted on July 26, 2018)

(C) 2018 Totem Publisher, Inc. All rights reserved.

\section{Introduction}

Hydrogen is a clean energy and an important chemical raw material. It has been widely applied in aerospace, fuel cell, metal smelting, chemical synthesis, and other fields [1]. At present, the hydrogen sensors used in the market are mainly electrochemical sensors, and a high-performance hydrogen sensor cannot be separated from the test and performance analysis of hydrogen sensor data in early stages. Therefore, a set of data acquisition and processing systems for hydrogen sensing materials is of great significance for the practical applications of hydrogen sensors [2].

\section{The Working Principle and Hardware Design of the System}

\subsection{The MCU Minimum System}

The data acquisition and processing system under the hydrogen sensor mainly includes four parts: signal acquisition circuit, signal conversion circuit, signal transmission circuit, and signal compensation and processing of upper computer. The principle diagram is illustrated in Figure 1. The signal acquisition circuit is mainly used to collect the resistance signal of the hydrogen sensor; the circuit signal conversion circuit transforms the resistance signal into the current signal and then turns to the voltage signal; the signal transmission circuit is used to upload the processed data to the upper computer; and the signal compensation and processing part of the upper computer mainly realizes the temperature compensation and number.

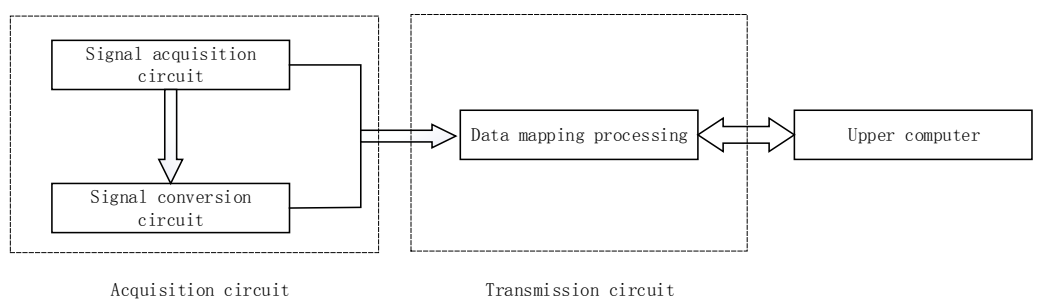

Figure 1. System block diagram

\footnotetext{
* Corresponding author.

E-mail address: yang.yj@ntu.edu.cn
} 


\subsection{The Signal Acquisition Circuit of the System}

The hydrogen sensing material for the system is a material with high voltage requirements and requires constant voltage. Therefore, the response of hydrogen induced material to hydrogen is manifested in the change of material resistance, and the current in the acquisition circuit reflects the change. The schematic diagram of the acquisition circuit is shown in Figure 2 first. The LM358 operational amplifier consists of two independent, high gain, and internal frequency compensation dual operational amplifiers [3]. It is suitable for single power supply with a wide range of power supply. It is also suitable for dual power mode. Under the recommended working conditions, the power current is independent of the power supply voltage. The system uses the "short" characteristic of the LM358 operational amplifier to put the voltage clamp in the $2 \mathrm{~V}$, in which the resistance R1 and the R2 make the operation and amplifier close closed, so the circuit is a closed loop state of negative feedback, thus ensuring the operation of the operational amplifier in the linear region. The voltage of the measured hydrogen induction material is always constant [4].

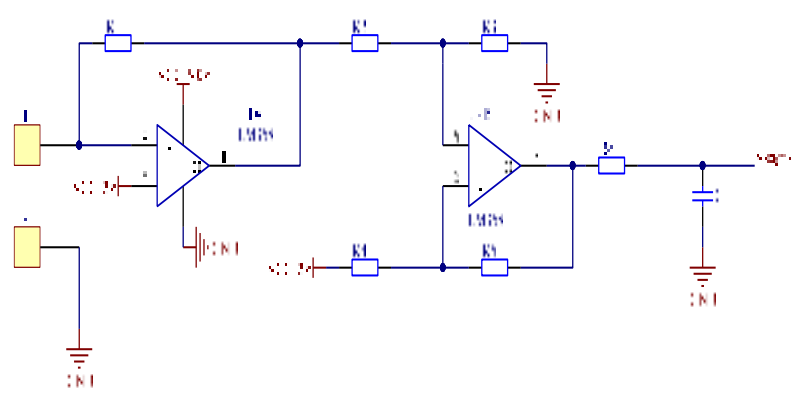

Figure 2. Signal acquisition circuit

The reaction of the signal change of the hydrogen sensor corresponds to the change of the resistance, while the hydrogen induction material requires constant voltage and the resistance value of the input resistance is within $200 \mathrm{~K}$ Omega. To overcome the adverse effects of all kinds of noise, it is necessary to amplify the signal, so the signal is amplified in the second stage of the signal acquisition circuit. Since the second stage is a phase input amplifying circuit, the input of the resistance is infinite, so it fully satisfies the requirement of the resistance change of the hydrogen gas material [5].

The third stage of the signal acquisition circuit makes use of the differential amplifier circuit to realize the conversion of the signal. This facilitates the AD sampling of the data, in which the signal V-Sigel is the corresponding voltage sampling and the $\mathrm{R}$ is the measured material resistance [6-7].

\subsection{The Signal Conversion Circuit of the System}

In the signal acquisition circuit of the system, the obtained resistance is mapped to the $0.0-100.0 \%$ according to the maximum and minimum value set by the host computer, and the sampling quantization value is obtained with a precision of $0.1 \%$. As shown in Figure 2, in the system signal conversion circuit, the system hardware master control STM32 has the PWM output function. The PWM+RC filter can be used to achieve the DAC output, thus saving the cost [7-8]. The signal conversion circuit is shown in Figure 3.

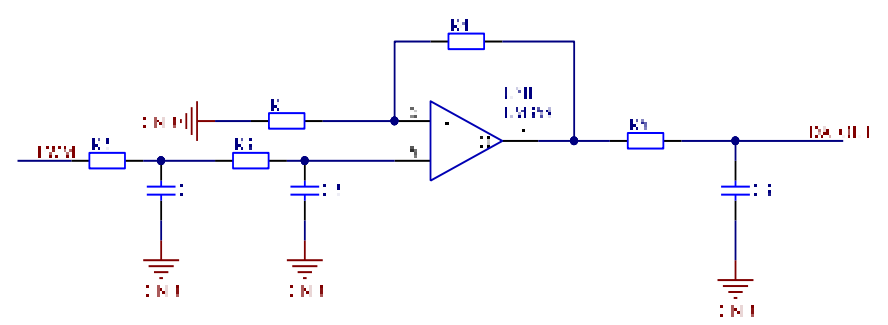

Figure 3. Signal conversion circuit

A certain period is actually the essence of PWM and the high-level adjustable duty cycle square wave. The PWM waveform of the actual circuit is shown in Figure 3, where the PWM waveform can be expressed as a piecewise function [9]. 


$$
f(t)= \begin{cases}V_{H}, & k N T \leq t \leq n T+k N T \\ V_{L}, & k N T+n T \leq t \leq N T+k N T\end{cases}
$$

Among them: $T$ is the basic period of counting pulses in the single-chip microcomputer, $N$ is the count pulse number of a cycle of PWM wave, $n$ is the count pulse number of high level in a cycle of PWM wave, $V_{H}$ and $V_{L}$ are the high and low voltage values of PWM wave, $k$ is the harmonic frequency, and $t$ is time. Formula (1) is expanded into a Fourier series, and then formula (2) is obtained.

$$
\begin{aligned}
f(t) & =\left[\frac{n}{N}\left(V_{H}-V_{L}\right)\right]+2 \frac{V_{H}-V_{L}}{\pi} \sin \left(\frac{n}{N} \pi\right) \cos \left(\frac{2 \pi}{N T} t-\frac{n \pi}{N} k\right) \\
& +\sum_{k=2}^{\infty} 2 \frac{V_{H}-V_{L}}{k \pi}\left|\sin \left(\frac{n \pi}{N} k\right)\right| \cos \left(\frac{2 \pi}{N T} k t-\frac{n \pi}{N} k\right)
\end{aligned}
$$

The first square brackets are DC components, the second ones are the harmonic components, and the third ones are the higher harmonic components. The DC component of $\mathrm{N}$ has a linear relationship with $\mathrm{N}$, and the DC component changes from $V_{L}$ to $V_{L}+V_{H}$ as $n$ changes from 0 to $N$. This is exactly what the voltage output DAC needs. Therefore, if the harmonics outside the DC component can be filtered out, the conversion from the PWM wave to the voltage output DAC can be obtained, that is, the PWM wave can be demodulated by a low pass filter. The magnitude and phase angle of the second terms in $\mathrm{n}$ are related to the frequency of $1 /(N T)$, which is actually the output frequency of PWM. The frequency is the basis for the design of low pass filters. If we can filter out the one harmonic, the high-order harmonics should not exist. Through the above understanding, we can obtain the resolution of PWM DAC. The formula is as follows:

The resolution is $=\log _{2} N$; suppose that the minimum change of $N$ is 1 ; when $N=256$, the resolution is 8 bits. The timer of STM32 is all 16 bits. It can easily achieve higher resolution. The higher the resolution, the slower the speed [10]. The DAC resolution of the system is 8 bits. Under the condition of 8-bit resolution, it is required that the influence of the one harmonic on the output voltage does not exceed the accuracy of $1 \mathrm{bit}$, that is, $3.3 / 256=0.01289 \mathrm{~V}$. Assuming that $V_{H}$ is $3.3 \mathrm{~V}$ and $V_{L}$ is $0 \mathrm{~V}$, the maximum value of one harmonic is $2 \times 3.3 / \mathrm{PI}=2.1 \mathrm{~V}$, which requires our RC filter circuit to provide at least $-20 \lg (2.1 / 0.01289)=-44 \mathrm{~dB}$ attenuation. The fastest counting frequency of the STM32 timer is $72 \mathrm{MHz}$. When 8 is the resolution, the PWM frequency is $72 \mathrm{M} / 256=281.25 \mathrm{kHz}$. If the first order RC filter is applied, the cut-off frequency is $1.77 \mathrm{kHz}$. If the second order RC filter is applied, the cut-off frequency is $22.34 \mathrm{kHz}$.

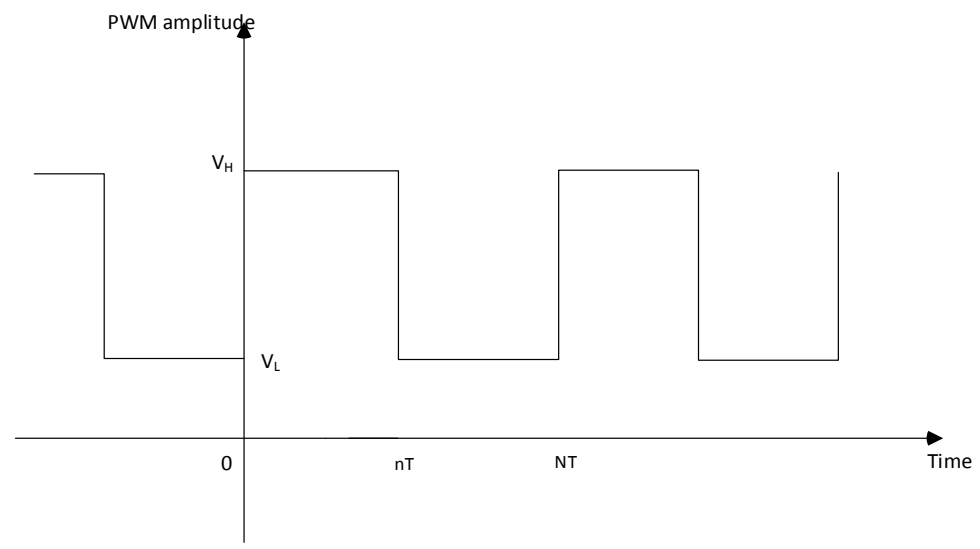

Figure 4. Pwm waveform

\section{Software Design of the System}

\subsection{Software Design of Acquisition End}

The program is written in the KEIL software and used in the C language. The software flow chart of the acquisition end is shown in Figure 5. After the software is started, the serial port, the AD collection, the FLASH reading and writing command, and the internal peripherals of the STM32 are initialized first. After the initialization is completed, the parameters of the data are set by reading the FLASH inside the STM32 [11]. If the current is an empty command, the upper computer waits to communicate with the host computer software until the host computer sends the basic set parameters to the 
acquisition end and the upper computer root, according to the collected data for temperature compensation processing. The parameters are set to the acquisition end. If the parameters are finished, the acquisition end needs to store the set parameters into the FLASH inside the STM32, and the next data collection and data processing according to the parameters are set. The parameters in the FLASH within the STM32 are stored as the next data acquisition finger until the host computer changes the setting parameters again [12].

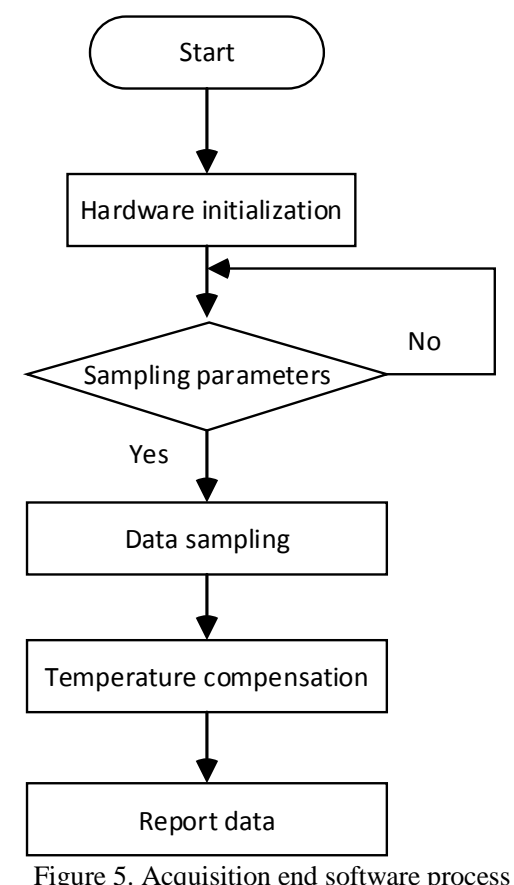

\subsection{Software Design of Upper Computer}

Visual Studio software is used as the development platform and written in C\# language. The upper computer software includes data display, sampling parameters setting, data storage, temperature complement setting, and so on. The host computer software and the acquisition terminal carry out the data communication through the serial port. Setting the parameters mainly includes setting the resistance mapping range, the analog output range, the serial output options, and the sampling rate. The data format is JSON. The temperature setting is in the range of $-15 \sim 55 \mathrm{C}$, with each 10 degrees being a gradient and a total of 7 gradients. The temperature compensation processing for the data is only needed to operate the host computer software, and the temperature compensation at the corresponding temperature can be realized. To facilitate the data collected and the corresponding setting parameters, the host computer software is convenient. The function of parameter preservation is completed [5]. Figure 6 is the actual interface diagram of the software.

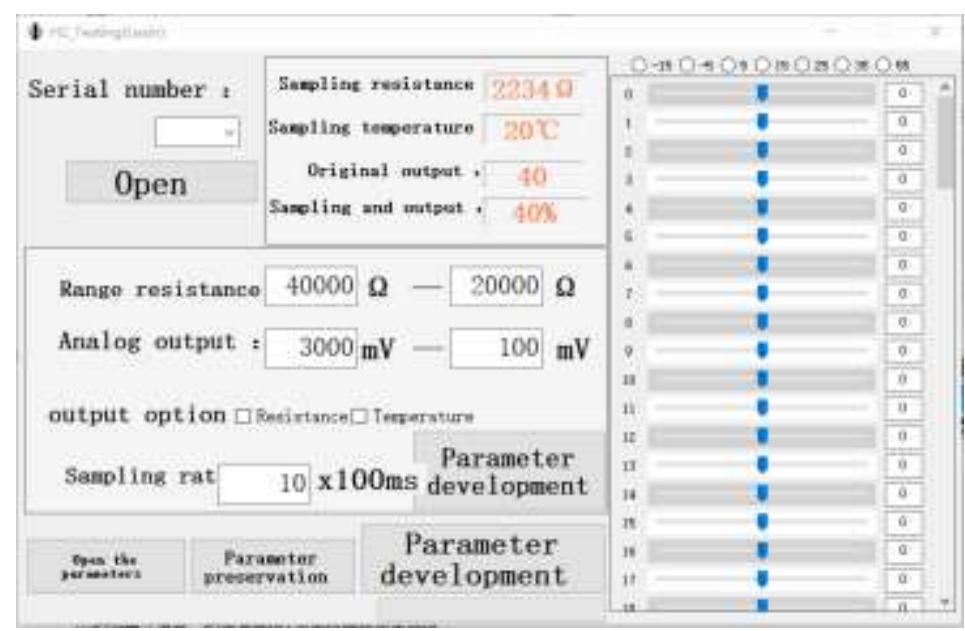

Figure 6. Software interface 


\section{The Test Results}

The hardware and software part of the whole system has been explained, and how to collect data is explained below. In the signal acquisition circuit of the system above, the hydrogen sensor's reaction to hydrogen shows the change of resistance, and for the final signal acquisition, the following relationship is met with the hydrogen sensor's resistance.

$$
V_{\text {singel }}=\left(1+\frac{R_{5}}{R_{4}}\right)\left(\frac{R_{3} / R_{2}}{1+R_{3} / R_{2}}\right)\left(2+\frac{2 R_{1}}{R}\right)-\frac{2 R_{5}}{R_{4}}
$$

In the upper third, $\mathrm{R}$ is the resistance of the hydrogen sensor to be measured, and V_singel is the final sampling voltage.

The Multisim is used to simulate the experiment, the variable resistance is used to simulate the change of the resistance of the sensor at the acquisition end, and the final voltage is sampled by the oscilloscope [13]. The experimental results are shown in Table 1.

Table 1. Experimental result

\begin{tabular}{|c|c|c|}
\hline Sensor resistance (Company: K $\Omega$ ) & Sampling voltage value(Company: V) & Experimental error(Company: V) \\
\hline 20.5 & 1.95 & 0.001 \\
\hline 80.2 & 0.49 & 0.008 \\
\hline 150 & 0.26 & 0.006 \\
\hline
\end{tabular}

The simulation results show that the final voltage sampling and resistance change satisfies 1 , while the actual hydrogen sensor test, combined with the temperature compensation function of the host computer, can fully meet the actual requirements of the measured hydrogen sensor for data processing.

\section{Conclusions}

In this paper, a set of systems for the theoretical analysis of hydrogen sensor data is realized. The system uses embedded technology and STM32 processor as the core, realizes the data acquisition of hydrogen sensors, assists the software to process and analyze the data, and lays a solid foundation for real-world applications of the late hydrogen gas sensor. The system is not limited to the analysis of the early data of hydrogen sensors: it can be used in the data analysis of the same kind of sensors and is also worthy of reference for other sensors [14].

\section{Acknowledgements}

This work was supported by the Open Research Project of Nantong Intelligent Information Technology Research Center (KFKT2017B05) of Nantong University and the First Phase Project of Jiangsu University Brand Specialty Construction Project (PPZY2015B135). In addition, it was also supported by the Graduate Student Innovation Program of Nantong University. The authors thank the three anonymous reviewers for their helpful suggestions.

\section{References}

1. Junaidi, L. Aba, and K. Triyana, "An Automatic Data Acquisition System for Optical Characterization of PEDOT:PSS-based Gas Sensor," in Proceedings of the $5^{\text {th }}$ Asian Physics Symposium, pp. 1790-1979, 2015

2. H. Zhan, M. Wang, B. Wang, J. Hu, and Z. Xue, "Research and Development of General Data Acquisition System based on Wireless Sensor Network Dynamic Network Technology," in Proceedings of 2016 IEEE International Conference of Online Analysis and Computing Science (ICOACS), pp. 310-314, 2016

3. G. Zhou, G. Xiong, F. Yu, et al. "Design and Implementation of High-Speed Real-Time Data Acquisition and Processing System based on FPGA," in Proceedings of International Conference on Social Science and Technology Education, 2016

4. M. Serbanescu, V. M. Placinta, O. E. Hutanu, and C. Ravariu, "Smart, Low Power, Wearable Multi-sensor Data Acquisition System for Environmental Monitoring," in Proceedings of 2017 10th International Symposium on Advanced Topics in Electrical Engineering (ATEE), 2017

5. W. U. Da-Zhong, Y. H. Peng, and N. W.Cao, "Design of Linear CCD Data Acquisition and Processing System based on ARM," Laser Journal, 2016

6. C. Lee, T. Kim, and S. J. Hyun, "A Sensor Network Query Processing System for Healthcare Data Acquisition in Dr. M," in Proceedings of International Conference on Data Engineering Workshops, pp. 78-83, 2016

7. Q. W. Wang, G. Y. Nan, J. H. Sun, J. F. Song, R. Guo, and J. Ma, "A Reference Architecture of Data Acquisition and Signal Processing of a Distributed Fiber-Optic Sensor System," Lecture Notes in Electrical Engineering, Vol. 334, pp. 723-730, 2015

8. M. A. B. Z. Abidin, M. Zolkapli, N. I. M. Noh, et al. "Data Acquisition for ISFET pH Sensor System by using Seeeduino 
Stalker as A Controller," in Proceedings of Control and System Graduate Research Colloquium, pp. 127-131, 2016

9. L. I. Ming, Y. Gao, J. S. Yang, and L. I. Xiao-Yu, "Study on Remote Data Acquisition System used in Time-Grating Displacement Sensor," Instrument Technique \& Sensor, 2017

10. Y. Y. Liu, L. I. Guo-Ping, G. K. Zuo, and L. I. Ya-Nan, "Design of Force Sensor Signal Acquisition and Processing System based on STM32," Transducer \& Microsystem Technologies, 2017

11. S. Mohanta, "A Labview based Data Acquisition System for Monitoring and Analysis of Vibration Signal," 2013

12. S. Florin, N. Sorin, N. Romeo, P. Anca, and F. Sergiu, "Data Acquisition and Processing with Fusion Sensors, used in Algorithms for Increasing Accuracy of Position Measurement of Mobile Robots," in Proceedings of International Workshop Soft Computing Applications, pp. 565-578, Springer, Cham, 2016

13. W. Wang, "Research on FPGA-based Data Acquisition and Processing System," Electronic Design Engineering, 2015

14. T. L. Brooks, X. Jiang, I. Mehr, D. J. Stoops, V. Jayakumar, M. G. Kim, H. Zheng, I. Ku, V. Chandrasekhar, and Y. L. Cheung, "Low-power Data Acquisition System and Sensor Interface," 2017

Shimin Wang is currently a graduate student in the School of Electronic Information at Nantong University. His main research interests include the Internet of things and embedded systems.

Yongjie Yang is a graduate of Nanjing University of Science and Technology and the dean of the School of Electronic Information at Nantong University. He has participated in national and provincial studies, and his main research direction is wireless communication.

Junjiao Zhang is currently a graduate student in the School of Electronic Information at Nantong University. Her main research interests include the Internet of things and embedded systems. 